\title{
Flow-Erleben in einem Computerspiel unter experimentell variierten Bedingungen ${ }^{1}$
}

\section{Flow experience in a computer game under experimentally controlled conditions}

\author{
Falko Rheinberg und Regina Vollmeyer
}

\author{
Adresse: \\ Institut für Psychologie der Universität Potsdam \\ Postfach 601553 \\ 14415 Potsdam \\ e-mail: rheinberg@,rz.uni-potsdam.de
}

\section{Summery}

The study presents a technique how to manipulate flow-experience via the computer game Roboguard. Under experimentally controlled conditions all parameters of the game and the situation were kept constant except the difficulty level the participants had to play on. Flow was assessed with the Flow Short Scale (FKS, Rheinberg et al., 2002). As predicted we received the highest Flow score on the medium/optimal level in comparison with an easy and difficult level $(d>1.0)$. We could not confirm the predicted negative effect of approval seeking goals (AGT, Hayamizu \& Weiner, 1991) on Flow experience. Instead all three AGT subscales correlated positively with the FKS-subscale absorption. Perhaps qualitative differences in initial motivation loose their influence if participants experience Flow under optimal conditions.

Key words: Flow-experience, Flow-Short-Scale, goal orientation, motivation.

\footnotetext{
${ }^{1}$ erschien 2003 in Zeitschrift für Psychologie, 4, 161-170.
} 
Berichtet wird über eine Möglichkeit, Flow-Erleben unter experimentell kontrollierten Bedingungen systematisch zu variieren. Dabei werden die Tätigkeit (das Computerspiel Roboguard) und die Situationsbedingungen konstant gehalten. Variiert wird lediglich die Schwierigkeitsstufe, auf der gespielt wird. Als abhängiges Maß wurde die Flow-Kurzskala (FKS, Rheinberg, Vollmeyer \& Engeser, 2002) verwandt. Es zeigten sich die vorhergesagten kurvilinearen Beziehungen zwischen Anforderungsstufe und Flow mit Effektstärken um 1 und größer. Zusammenhänge zwischen habitueller Zielorientierung (Hayamizu \& Weiner, 1991) und Flow zeigten sich nur bei den flow-auslösenden Schwierigkeitsstufen. Es gab keine negative Beziehung zwischen Zielorientierung und Flow, vielmehr korrelierten sowohl die learning- als auch die performance goal orientation positiv mit der Flowkomponente Absorbiertheit. Aus diesem Befund wird eine Arbeitshypothese zur Beziehung von Motivation und Flow hergeleitet, wonach unter optimalen Bedingungen Besonderheiten der Initialmotivation vielleicht dann keine Rolle mehr spielen, wenn die Person erst einmal im Flow-Zustand ist.

Stichworte: Flow-Erleben, Flow-Kurz-Skala, Motivation, Zielorientierung. 
Unter Flow-Erleben versteht man das (selbst-)reflexionsfreie Aufgehen in einer glatt laufenden Tätigkeit, die man trotz hoher Beanspruchung noch unter Kontrolle hat (Csikszentmihalyi, 1975/1999; Rheinberg, 2002). Csikszentmihalyi ist auf diesen Zustand gestoßen, als er Personen zu Besonderheiten und Anreizen von Aktivitäten befragte, die sie ohne erkennbare Belohnung hoch engagiert betrieben. In den retrospektiven Schilderungen dieser Personen fand Csikszentmihalyi immer wieder sechs bis neun Komponenten, die die Besonderheiten dieses Zustandes ausmachen. Tabelle 1 führt die sechs wichtigsten Komponenten auf.

Die qualitative erste Forschungsphase wurde abgelöst durch Untersuchungen mit der sogenannten Experience Sampling Method (ESM, Csikszentmihalyi \& Larson, 1987; Csikszentmihalyi \& LeFevre, 1989). Hierbei werden die Untersuchungsteilnehmer im Tagesverlauf meist acht Mal von einem Signalgeber dazu aufgefordert, ihren momentanen Zustand und ihre Tätigkeit auf mitgeführten Skalen zu beurteilen. Der Vorteil dieser ESM liegt zweifellos darin, dass die Erhebung direkt an den Tätigkeitsvollzug herangerückt wird.

Es gibt bei diesen Untersuchungen allerdings den kritischen Punkt, dass die Autoren bei der Auswertung der Daten den Flow-Zustand ungeprüft immer dann als gegeben annehmen, wenn die Fähigkeitsselbsteinschätzungen den wahrgenommenen Anforderungen auf anspruchsvollem Niveau entsprechen (Balance bzw. Passung auf hohem Niveau). Damit wird von nur einer (wichtigen) Flow-Komponente auf das gesamte Konstrukt geschlossen (z. B. Csikszentmihalyi \& LeFevre, 1989; Moneta \& Csikszentmihalyi, 1996). Das ist insofern problematisch, als man aus der traditionellen Leistungsmotivationsforschung ja weiß, dass gerade unter herausfordernden Passungsbedingungen nicht nur positive Aufsuchungstendenzen, sondern auch Meidentendenzen angeregt werden können. Ob eher Aufsuchen oder Meiden angeregt werden, hängt entscheidend von der Motivausprägung der Person ab (Atkinson, 1957; Heckhausen, Schmalt \& Schneider, 1985). 
Inzwischen liegen einige Fragebögen vor, die Flow-Erleben nicht nur über eine, sondern über alle Komponenten dieses Zustandes erfassen. Novak, Hoffman und Young (1998) haben ein umfangreiches Inventar entwickelt, das für die Nutzung des Internets eingesetzt wird (75 Items). Jackson und Eklund (2002) arbeiten mit einer Skala, die das FlowErleben im Sport erfasst (36 Items). Remy (2000) hat einen Fragebogen zum Flow-Erleben bei der PC-Arbeit entwickelt (35 Items).

Eine Schwierigkeit längerer Flow-Skalen liegt darin, dass sie den aktuellen Tätigkeitsablauf spürbar unterbrechen. Das schränkt ihre Einsetzbarkeit ein. Von daher haben Rheinberg, Vollmeyer und Engeser (2002) eine Flow-Kurzskala (FKS) entwickelt, die mit zehn Flow- und drei Besorgnis-Items innerhalb einer Minute bearbeitbar ist. Die Items der FKS sind so formuliert, dass sie auf jedwede Aktivität passen. Von daher ist die FKS beliebig einsetzbar und auch mit der ESM kombinierbar.

Rheinberg et al. (2002) berichten zu dieser Skala einige Validitätsbefunde. Bei Psychologie Studierenden, die sich mit Übungsaufgaben auf ihre Statistikklausur vorbereiteten, klärte der Flow-Wert der FKS zusätzlich 5.3 \% der späteren Leistungsvarianz auf und zwar auch dann, wenn alle anderen signifikanten Leistungsprädiktoren (Mathematiknote, Statistikvorkenntnisse, Alter) vorher in die Regressionsgleichung eingeführt wurden. Der Befund ist inzwischen repliziert (Rheinberg, Engeser \&Vollmeyer, 2002). Bei einer anderen intellektuell herausfordernden Aufgabe, nämlich der PostkorbÜbung (Musch, Rahm \& Lieberei, 2001), zeigte sich, dass die erfolgszuversichtliche Komponente des Leistungsmotivs einen signifikanten Einfluss auf den FKS-Flow-Wert während der Übung hatte (standardisiertes $\beta=.47, p<.01$ ). Die misserfolgsängstliche Komponente des Leistungsmotivs beeinflusste dagegen den Besorgnis-Wert der FKS (standardisiertes $\beta=.34, p<.01$ ).

Was Personenunterschiede betrifft, so vermuten Csikszentmihalyi und Jackson (2000), dass die Zielorientierung einer Person einen Effekt auf das Flow-Erleben haben sollte. 
Personen, die im Sinne von Dweck und Leggett (1988) oder Nicholls (1984) ihre Fähigkeit demonstrieren wollen (performance goals), sollten weniger gut in den Flow-Zustand kommen als Personen, die bei herausfordernden Aufgaben etwas dazu lernen wollen (learning goals). Diese Annahme würde insofern Sinn machen, als der Gedanke daran, ob das spätere Handlungsergebnis anderen Personen die eigene Fähigkeit zeigen würde, eine verrichtungsferne Kognition ist, die das Aufgehen in der Tätigkeit beeinträchtigen müsste. Ein Versuch, diese durchaus plausible Annahme z. B. im Sport empirisch klar zu belegen, ist allerdings fehlgeschlagen (Jackson, Kimiecik, Ford \& Marsh, 1998).

\section{Zielsetzung der Arbeit}

Insgesamt präsentiert sich das Konzept des Flow-Erlebens als interessanter und reizvoller Forschungsgegenstand, zu dem aber noch erheblicher Klärungsbedarf besteht (Rheinberg, 2002). Insbesondere auf forschungsmethodischer Ebene erscheinen Verbesserungen nötig und möglich. Wir haben versucht, in einem Punkt eine Verbesserung zu erreichen. Es geht um den Versuch, Flow unter experimentell kontrollierten Bedingungen zu erzeugen.

Insbesondere die Flow-Erhebungen mit der ESM-Technik haben zweifellos den Vorteil, ökologisch hoch valide zu sein. Die Daten stammen ja direkt aus dem Alltag - vom Arbeitsplatz und aus der Freizeit (z.B. Csikszentmihalyi \& LeFevre, 1989; Pfister, 2002; Schallberger \& Pfister, 2001;), ja sogar direkt aus der Felswand bei Kletterern (Aellig, 2002). Die hohe ökologische Datenvalidität bringt allerdings auch einige Unkontrollierbarkeiten mit sich. Letzteres fällt dann ins Gewicht, wenn man den Flow-Zustand im Detail untersuchen will, insbesondere hinsichtlich seiner Korrelate und Erscheinungsformen sowie hinsichtlich seiner Folgen.

In einer ESM-Studie mit Studierenden fanden wir beispielsweise, dass die höchsten Flow-Werte (FKS) beim „Sex/Intimitätenaustausch“, beim „Sport“ und beim „Musizieren“ auftraten. Die niedrigsten Flow-Werte traten dagegen beim „Warten“ und „Sinnieren/trüben Gedanken nachhängen“ auf (Rheinberg et al., 2002). Das erscheint durchaus plausibel, bringt 
jedoch Probleme für Detailanalysen. Zwischen Sex, Musizieren und Sport gibt es trotz der Gemeinsamkeit im Flow sicherlich eine Vielzahl von Verrichtungs- und Erlebnisbesonderheiten, in denen sie sich deutlich von einander unterscheiden. Kontrastiert man jetzt Aktivitäten mit unterschiedlichen Flow-Werten, wird man je nach einbezogenen Aktivitäten mal diesen und mal jenen Unterschied finden, ohne zu wissen, ob das wirklich charakteristisch für den Flow-Zustand oder für die jeweils einbezogene Tätigkeit ist. Und selbst, wenn man das wüsste, ergibt sich für Detailanalysen das Problem, dass die unterschiedlichsten flow-irrelevanten Aktivitätsbesonderheiten dasjenige überlagern können, was man als flow-spezifisch untersuchen möchte.

Ideal wäre von daher eine Möglichkeit, Flow-Erleben innerhalb derselben Aktivität und unter standardisierten Bedingungen verschieden stark anregen zu können. Sofern das gelingt, hätte man den Einfluss irrelevanter Merkmale unterschiedlicher Tätigkeiten und Ausführungssituationen ausgeschaltet. Damit könnte man ungestörter den jeweils interessierenden Erlebnis- und Verhaltensbesonderheiten des Flow-Erlebens nachgehen. Die Frage ist allerdings, ob sich Flow unter experimentell kontrollierten Bedingungen überhaupt induzieren lässt. Dieser Frage sind wir nachgegangen mit dem Ziel, eine standardisierbare Methode zur Manipulation von Flow-Zuständen zu finden.

\section{Das Computerspiel Roboguard}

Aufgrund informeller Beobachtungen und Selbstberichte (z. B. Schubert, 1986) erschien uns ein Computerspiel geeignet, Flow-Zustände unter kontrollierten Bedingungen zu manipulieren. Hier bietet sich die Möglichkeit, alle Bedingungen der Aufgabe und der Situation konstant zu halten und lediglich einen relevanten Parameter zu verändern, nämlich die Anforderung/Schwierigkeit des Spiels. Gemäß dem ursprünglichen Flow-Kanalmodell von Csikszentmihalyi (1975/1999) sollten bei geeigneten Rahmenbedingungen die FlowWerte mit wachsenden Anforderungen zunächst bis zu einem optimalen Beanspruchungsgrad steigen, um dann bei weiterer Anforderungssteigerung wieder abzufallen. Sollte sich ein 
solcher kurvilinearer Verlauf der Flow-Werte nachweisen lassen, hätte man eine Möglichkeit gefunden, den Flow-Zustand unter kontrollierten Bedingungen zu manipulieren, indem man bei einem geeigneten Computerspiel die Anforderungen systematisch variiert.

Benötigt wurde dazu ein Computerspiel, das (1) bei passender Schwierigkeitsstufe Probanden hinreichend stark absorbiert. (2) Weiterhin sollte das Spiel keine offensivaggressiven Akte von den Spielern verlangen. (3) Schließlich müsste man den Schwierigkeitsgrad des Spiels willkürlich einstellen können, (d. h. das Spiel dürfte nicht selbstadaptiv sein und man müsste in sein Programm eingreifen können). (4) Ernsthafte Versagensbefürchtungen sollten allerdings keine Rolle spielen, weil dann nämlich Interaktionen mit Personenvariablen, insbesondere mit der Richtung des Leistungsmotivs zu erwarten wären. Solche Komplikationen wollten wir in der jetzigen Forschungsphase aber möglichst noch ausschließen.

Das hier scheinbar nahe liegende Spiel Tetris erweist sich als weniger geeignet, weil bei diesem sehr bekannten Spiel sich einige Probanden auf den niedrigen Anforderungsstufen die Aufgabenschwierigkeit selbst hochfahren, indem sie den Start ihres Eingreifens hinauszögern. Entsprechend ungünstig waren die Erfahrungen bei dem Versuch, FlowErleben mithilfe von Tetris zu variieren (Remy, 2000).

Wir wählten das weniger bekannte Spiel Roboguard. Bei diesem relativ einfach aufgebauten Spiel muss man ein Raumschiff steuern, das ständig vor „feindlichen“ Raketen fliehen muss. Erreichen diese Raketen das eigene Raumschiff, wird es zerstört und man muss neu starten. Das Ziel des Spiels besteht also darin, möglichst lange erfolgreich zu fliehen, d. h. nicht zerstört zu werden. Die Spielschwierigkeit ist (a) durch die Geschwindigkeit der Flugkörper sowie (b) die Zahl der verfolgenden Raketen zu variieren.

Neben dem eigenen Raumschiff und den verfolgenden Raketen gibt es noch einen Meteor und den Roboguard. Mit genügend Übersicht und Geschick kann man eine verfolgende Rakete nahe an beide Flugkörper locken, was zur Zerstörung dieser Rakete, 
mithin zur Verlängerung der eigenen Unversehrtheit beiträgt. Dabei muss man allerdings darauf achten, dass man nicht selber dem Meteor zu nahe kommt, weil das wiederum zur Selbstzerstörung führen würde. Überdies muss man natürlich darauf achten, dass man bei seinen Anlockversuchen nicht von einer der anderen verfolgenden Raketen eingeholt wird. Das wird mit steigender Zahl und Geschwindigkeit der Flugkörper immer schwieriger. (Durch geeignete Veränderung des Programms wurde es möglich, hierzu beliebige Schwierigkeitsstufen zu erzeugen. Das Programm kann bei den Autoren angefordert werden.) Vorstudie

Fragestellung

Zunächst musste geklärt werden, ob es bei diesem Spiel überhaupt zu einer systematischen Variation der Flow-Indikatoren kommt, wenn man die Aufgabenanforderungen verändert. Sollte das der Fall sein, wäre mit Blick auf nachfolgende Experimente festzustellen, in welchem Schwierigkeitsbereich das Maximum der Flow-Werte liegt.

\section{Methode}

Probanden. 18 Studierende nahmen an dem Experiment ohne Vergütung teil. Die Probanden waren überwiegend interessiert an Computerspielen.

Untersuchungsmaterial. Der Flow-Zustand wurde mit der FKS erfasst (s. Anhang).

Diese Skala liefert einen Gesamtwert (Cronbachs $\alpha$ um .90) sowie zwei faktorenanalytisch begründbare Unterskalen, nämlich Absorbiertheit sowie glatter automatisierter Verlauf (Rheinberg et al., 2002). Für das Ziel der Vorstudie genügten Analysen mit dem Gesamtwert. Die Einschätzung der Anforderungen erfolgte auf einer Neun-Punkte-Skala von: „Für mich persönlich sind die Anforderungen zu gering“ (1) über „,gerade richtig“(5) bis ,zu hoch“ (9).

Versuchsablauf. Eingesetzt wurden zehn Schwierigkeitsstufen des Spiels in fester Reihenfolge und zwar so, dass sich die sehr leichten und sehr schwierigen Stufen nicht häuften (Reihenfolge: 1, 3, 4, 8, 2, 10, 5, 7, 9, 6). Jeder Durchgang dauerte vier Minuten. 
Unmittelbar nach jedem Durchgang bearbeiteten die Probanden die FKS und schätzten den Schwierigkeitsgrad des Durchgangs ein (s. Anhang).

\section{Ergebnisse}

Wie erwartet, zeigte sich ein linearer Trend beim Zuwachs der erlebten Anforderung über die Schwierigkeitsstufen des Spiels hinweg (Test auf Linearität: $F(1,17)=36.17, p<.001$ ). Abbildung 1 zeigt den Verlauf der erlebten Schwierigkeit über die Schwierigkeitsstufen hinweg (benachbarte Schwierigkeitsstufen sind paarweise zusammengefasst).

Insgesamt hatten wir das Spiel für unsere computerspielinteressierten Probanden wohl etwas zu leicht eingestellt. Jedenfalls blieben bis zur Schwierigkeitsstufe $8(M=4.39, S D=$ 0.85) die subjektiven Einschätzungen im Mittel unterhalb der Skalenmitte (5.00 für die optimale Beanspruchung „gerade richtig“ $)$. Die Stufe $9(M=6.61, S D=1.29)$ und Stufe 10 $(M=7.89, S D=2.08)$ liegen dann aber klar oberhalb der optimalen Beanspruchung.

Nach diesen Daten sollte der Flow-Wert der FKS bis zur Stufe 8 steigen, um nach Überschreiten der optimalen Beanspruchung wieder zu fallen. Tatsächlich wird auf Stufe 8 der höchste Flow-Wert in diesem Spiel erreicht $(M=4.81, S D=1.27)$. Auf Stufe $9(M=4.42$, $S D=1.25)$ und Stufe $10(M=3.12, S D=1.49)$ fällt er dann deutlich ab. Über alle Stufen hinweg lässt sich der quadratische Trend der Flow-Werte absichern, $F(1,17)=16.68, p<$ .001. Abbildung 1 zeigt den Verlauf der Flow-Werte für die zusammengefassten Paare von Schwierigkeitsstufen. Besorgnisse und ernsthafte Misserfolgsbefürchtungen spielen im Mittel hier keine oder nur eine geringe Rolle. Mit $M=1.79(S D=0.94)$ liegt der Besorgnis-Wert eine bis zwei Standardabweichungen unterhalb der Werte, die aus anderen Leistungssituationen bekannt sind (Rheinberg et al., 2002).

Fazit

Beim Spiel Roboguard scheint es möglich, über die Manipulation der Anforderungen den Flow-Wert der FKS systematisch zu variieren. Es wurden Effektstärken bis zu $d=1.22$ festgestellt (Stufe 8 vs. Stufe 10). Von daher ließen sich die Effekte auch schon bei geringer 
Stichprobengröße $(N=18)$ absichern. Beim Vergleich mit anderen Stichproben übertreffen die jetzigen Flow-Werte auf den Stufen 7 und 8 die üblichen FKS-Mittelwerte. Bei Untersuchungen einer spezifischen Tätigkeit auf Stichprobenebene haben wir bislang lediglich bei illegalen Graffiti-Sprayern einen höheren Flow-Mittelwert gefunden (Rheinberg et al., 2002).

Dass die Besorgnis-Werte so niedrig liegen, war erhofft. Damit ist natürlich nicht ausgeschlossen, dass bestimmte Personen sich sogar in dieser Spielsituation mehr Sorgen über ihr Abschneiden machen als andere (s. unten). Gleichwohl werden diese Unterschiede auf insgesamt niedrigem Besorgnisniveau auftreten. Das Spiel hat in der Vorstudie das geleistet, was es sollte.

Flow in Abhängigkeit von Anforderung und Zielorientierung

\section{Fragestellung und Hypothesen}

Nachdem die flow-vermittelnden Anforderungsstufen bekannt sind, können wir jetzt mit einer größeren Stichprobe gezielter vorgehen, um (1) den Flow-Effekt der Anforderungsvariation zu replizieren und (2) theoretisch interessierenden Beziehungen zwischen Personenmerkmalen und Flow-Erleben nachzugehen. Dabei können wir uns jetzt auf drei Schwierigkeitsgrade beschränken (leicht, mittel/optimal, schwer). Im Einzelnen gehen wir zwei Vorhersagen nach:

Hypothese 1: Die Flow-Werte haben einen kurvilinearen Verlauf über ansteigende Spielanforderungen mit einem Maximum bei mittleren/optimalen Anforderungen. Diese Hypothese ergibt sich aus dem Flow-Kanalmodell von Csikszentmihalyi (1975/1999) und stützt sich auf den entsprechenden Befund der Vorstudie (s. o.).

Hypothese 2: Eine Zielorientierung, die Anreize des Tätigkeitsvollzuges betont (z. B. eine learning goal orientation sensu Dweck \& Leggett, 1988), wirkt sich förderlich auf FlowErleben bei der Tätigkeit aus. Dagegen sollte eine Zielorientierung, die verrichtungsirrelevante Anreize betont (z. B. bestimmte Formen einer performance goal 
orientation), sich beeinträchtigend auf Flow-Erleben auswirken. Diese Hypothese folgt der Argumentation von Csikszentmihalyi und Jackson (2000). Sie erscheint plausibel, da alles, was die Aufmerksamkeit von der Tätigkeitsverrichtung weg lenkt, das Aufgehen in der Tätigkeit stören müsste. Trotzdem konnte in Felduntersuchungen diese Hypothese nicht bestätigt werden (Jackson et al., 1998; Stein, Kimiecik, Daniels \& Jackson, 1995).

Wir führen letzteres (a) auf die nur eingeschränkten Kontrollmöglichkeiten in Felduntersuchungen zurück, wodurch schwächere Effekte verwischt werden. Unabhängig davon ist (b) unklar, ob die bislang getroffene Grobunterscheidung zwischen learning vs. performance goals genau das trifft, worauf es ankommt. So finden Harackiewicz und Mitarbeiter (z. B. Barron \& Harackiewicz, 2001) wiederholt positive Einflüsse der performance goal orientation auf Lernen und Lernleistung. Das widerspricht klar den bislang akzeptierten Annahmen von Dweck und Leggett (1988), wonach die learning, nicht aber die performance goal orientation positive Lern- und Leistungseffekte haben sollte (zur aktuellen Kontroverse, s. Sansone \& Harackiewicz, 2001).

Mit Blick auf Flow sollte die Vorliebe für neue Herausforderungen und die Freude an der Kompetenzsteigerung (learning goal orientation) das Aufgehen in einer leistungsthematischen Tätigkeit fördern. Bei der performance goal orientation ist jedoch danach zu unterscheiden, ob jemand auf verrichtungsirrelevante Anreize zielt (z. B. durch gute Resultate beliebt zu werden = approval seeking goal, Hayamizu \& Weiner, 1991) oder auf leistungskontingente Anreize achtet (z. B. leistungsabhängige Aufstiegschancen für weitere Leitungsaktivitäten zu gewinnen = performance advance goals, Hayamizu \& Weiner, 1991). Im letzteren Fall hätten wir nach Heckhausen (1989 durch die Gleichthematik von Handlung, Ergebnis und Folgen eine intrinsische (Leistungs-) Motivation, die nicht notwendig das Aufgehen in der Tätigkeit stören müsste. Im Fall der Anerkennungssuche (approval seeking goals) müssten jedoch die Gedanken an die Fremdbewertung eigenen Tuns und das Bemühen um optimierte Selbstpräsentation das reflexionsfreie Aufgehen in der 
Tätigkeit, also Flow, behindern. Von daher erwarten wir unter Hypothese 2 nur für den letzteren Fall, also die approval seeking goals, nicht aber für die performance advance goals einen ungünstigen Effekt auf Flow-Erleben.

\section{Methode}

Probanden. 46 Studierende (davon 37 weiblich) nahmen an dem Experiment teil. Sie erhielten entweder eine Vergütung von 5 Euro oder ein Testat über eine Versuchspersonenstunde.

Untersuchungsmaterial. Das verwandte Computerspiel Roboguard ist in der Vorstudie schon beschrieben worden. Das Flow-Erleben wurde mit der FKS (Rheinberg et al., 2002, s. Anhang) erfasst. Die Reliabilität der Skala lag in den fünf Durchgängen zwischen Cronbachs $\alpha=.80$ und .90. Die Zielorientierung wurde mit dem Achievement Goal Tendency Questionnaire (AGT, Hayamizu \& Weiner, 1991) erhoben, weil dieses Instrument eine Aufspaltung der performance goal Komponente vornimmt, die uns mit Blick auf FlowErleben wichtig erscheint. Der AGT unterscheidet nämlich zwischen approval seeking goals („Ich lerne, weil ich von meinen Freunden anerkannt sein möchte.“) und performance advance goals (,Ich lerne bzw. studiere, weil ich einen guten Job haben möchte.“). Gemäß Hypothese 2 erwarten wir nur von der ersten Komponente, nicht aber von der zweiten, dass sie flow-beeinträchtigend wirkt. Neben diesen beiden performance goals erfasst der AGT die Orientierung an learning goals („Ich lerne bzw. studiere, weil ich es genieße festzustellen, wie weit ich mich verbessert habe."). Entsprechend der Hypothese 2 erwarten wir von dieser Skala, dass sie einen positiven Effekt auf die Flow-Werte der FKS hat.

Der AGT-Fragebogen wurde in einer deutschen Version mit 15 Items gegeben. Die Reliabilität der Skalen learning goals war mit Cronbachs $\alpha=.64$ suboptimal, aber noch akzeptabel. Die beiden anderen Skalen waren mit $\alpha=.79$ (performance advance goals) bzw. $\alpha=.82$ (approval seeking goals) hinreichend reliabel. Die Skalen learning goals und performance advance goals erwiesen sich mit $r=.54(p<.01)$ als erheblich korreliert, was 
mit Blick auf die aktuelle Kontroverse um die Wirkung von performance vs. learning goals (Sansone \& Harackiewicz, 2001) nicht uninteressant ist. Als unabhängig zeigte sich lediglich die Skala approval seeking goals.

Versuchsablauf. Nach einer zweiminütigen Testphase gab es fünf Spieldurchgänge zu je fünf Minuten. Die Durchgänge 1, 3 und 5 wurden auf einer optimal angepassten mittleren Schwierigkeitsstufe gespielt, die zu einem erhöhten Flow-Wert führen müsste. Durchgang 2 wurde auf der sehr hohen Schwierigkeitsstufe 9 (die Stufen sind identisch mit denen der Vorstudie), Durchgang 4 auf der sehr niedrigen Schwierigkeitsstufe 2 gespielt. Auf Grund der Stichprobenrekrutierung (Teilnahme wurde diesmal vergütet, s. oben) nahmen nicht nur computerspielinteressierte, sondern auch spielunerfahrene Probanden teil. Wegen der unterschiedlichen Spielerfahrung musste der Schwierigkeitsgrad der optimalen Schwierigkeitsstufe individuell angepasst werden. Nach ihren Leistungen in der vorgeschalteten Testphase wurde den Probanden dazu die Stufe 6, 7 oder 8 aus der Vorstudie als optimale Schwierigkeitsstufe zugewiesen. Unmittelbar nach jedem Durchgang wurde das Flow-Erleben mit der FKS erhoben. Vor dem Experiment wurde die Zielorientierung mit dem Achievement Goal Tendency Questionnaire (AGT) von Hayamizu und Weiner (1991) erfasst. Einschließlich mündlicher Einführungen dauerte das Experiment ca. 50 Minuten.

Ergebnisse

Flow-Effekte der Anforderungsstufe. Die Durchgänge 1, 3 und 5 waren auf einer individuell angepassten optimalen Anforderungsstufe gespielt worden. Der Durchgang 2 war mit Schwierigkeitsstufe 9 sehr schwer, Durchgang 4 mit Schwierigkeitsstufe 2 sehr leicht. Wir hatten für die optimale Anforderungsstufe höhere Flow-Werte vorhergesagt als für die beiden nicht gepassten Anforderungsstufen. Abbildung 2 zeigt die Flow-Werte der FKS in den fünf Durchgängen. 
Der Mittelwertsunterschied der FKS-Werte zwischen den fünf Durchgängen ist mit $F(4,42)=26.11, p<.001$, hoch signifikant. Auf der optimalen Schwierigkeitsstufe $(1,3$ und 5) werden jedes Mal hohe FKS-Werte erzielt, bei sehr leichten bzw. sehr niedrigen Schwierigkeitsstufen sind die FKS-Werte jedes Mal hoch signifikant niedriger (alle entsprechenden Einzelvergleiche $p<.001$ ). Die Effektstärken liegen zwischen $d=1.27$ und $d$ = 1. 69. Die Effekte sind damit als stark zu klassifizieren.

Es sieht so aus, als ob die Flow-Werte bei optimaler Passung mit der Spieldauer zunehmen. Das würde Sinn machen, weil man mit zunehmender Vertrautheit flüssiger spielen kann. Die Überprüfung eines linearen Trends über die Durchgänge 1, 3 und 5 bestätigt diese post hoc-Vermutung, $F(1,45)=8.94, p=<.005$.

Fasst man die drei optimalen Schwierigkeitsstufen zusammen, zeigt sich deutlich der in Hypothese 1 vorhergesagte kurvilineare Verlauf der Flow-Werte über den drei Schwierigkeitsstufen. Abbildung 3 zeigt den Verlauf der beiden Flow-Unterskalen glatter, automatisierter Verlauf und Absorbiertheit sowie der Skala Besorgnis. Man sieht deutlich, dass beide Unterskalen unterschiedlich auf zu geringe Anforderungen reagieren. Kontrastiert man die geringe mit der optimalen Anforderungsstufe, so ist bei der Skala Absorbiertheit die Effektstärke mit $d=2.34$ mehr als doppelt so hoch wie bei der Skala glatter automatisierter Verlauf $(d=.94)$. Durch Unterforderung wird also in erster Linie die Absorbiertheit während des Spiels beeinträchtigt, während der glatte Verlauf dadurch weniger gestört wird. Das erscheint plausibel und ist vielleicht auch auf Alltagsbedingungen übertragbar.

Die Besorgnis-Werte der FKS sind wieder sehr niedrig und unterhalb der Werte, die wir bislang in anderen Untersuchungen gefunden haben. Mit einem Mittel von $M=1.73$ (SD $=1.04)$ entsprechen sie ziemlich genau dem Wert aus der Vorstudie $(M=1.79, \mathrm{SD}=0.94)$.

Hypothese 2 sagt einen positiven Zusammenhang zwischen einer learning goal orientation und Flow-Erleben vorher sowie einen negativen Zusammenhang zwischen approval goal orientation und Flow-Erleben. Hinsichtlich der performance advance goals 
waren die Vorhersagen nicht gerichtet. Wir erwarteten jedenfalls keinen negativen Zusammenhang mit Flow. Besorgnis sollte in dieser Spielsituation keine Rolle spielen, weswegen diesbezüglich auch keine Hypothesen formuliert wurden. Tabelle 2 zeigt die Korrelationen für die Durchgänge auf den optimalen Schwierigkeitsstufen 1, 3 und 5. (Bei den zu geringen und zu hohen Anforderungen hatten sich plausibler Weise keinerlei Zusammenhänge mit den drei Skalen der Zielorientierung ergeben, weswegen diese Schwierigkeitsstufen hier nicht weiter beschrieben werden.)

Wir sehen zwei Überraschungen. (1) Es gibt keine negativen Zusammenhänge! Entgegen Hypothese 2 scheint auch die Tendenz zum approval seeking das Flow-Erleben beim Computerspiel nicht zu beeinträchtigen. (2) Überraschenderweise hat das approval seeking aber einen signifikanten Zusammenhang mit der FKS-Komponente Besorgnis $(r=$ $.36, p<.05$ ). Zwar ist insgesamt die Besorgnis in diesem Spiel auf niedrigem Niveau (s. o.), aber wenn sich jemand überhaupt Sorgen macht, hier schlecht abzuschneiden, dann scheint das mit seiner Orientierung zusammenzuhängen, durch Leistung Anerkennung und Wertschätzung von anderen Personen erlangen zu wollen (approval seeking). Wir hatten nicht gedacht, dass sich so etwas sogar in einem doch unwichtigen Computerspiel zeigen könnte und hatten deshalb keine Hypothese dazu formuliert.

Zwischen der FKS-Komponente glatter automatisierter Verlauf und den Zielorientierungen ergibt sich kein Zusammenhang, wohl aber bei der FKS-Komponente Absorbiertheit. Hier findet sich die vorhergesagte Beziehung mit der learning goal orientation $(r=.34, p<.05)$. Allerdings zeigt sich hier auch bei der performance advance goal Orientierung ein hoch signifikanter Zusammenhang $(r=.40, p<.01)$. Letztere hat mit $r$ $=.32(p<.05)$ auch als einzige einen schwachen Zusammenhang mit dem Flow-Gesamtwert der FKS. Dabei hatten wir für diese Zielorientierung ja lediglich vermutet, dass sie - als (nach Heckhausen, 1989) intrinsisch leistungsmotivationale Orientierung - keinen negativen Zusammenhang mit Flow haben sollte. 


\section{Diskussion}

Ziel der vorliegenden Arbeit war es, eine Verfahren zu schaffen, Flow-Erleben unter experimentell kontrollierbaren Bedingungen durch die Variation eines einzigen Parameters zu manipulieren. Akzeptiert man die FKS als adäquates Flow-Maß, so ist das gelungen. Über die Variation der Schwierigkeitsstufen ergaben sich Effekte für die FKS-Werte, die als stark zu klassifizieren sind (d bis maximal 2.34 bei der Absorbiertheit). Da die bislang vorliegenden Validitätsbefunde dafür sprechen, dass das verwendete Flow-Maß (FKS) tatsächlich das erfasst, was es soll - nämlich das (selbst-) reflektionsfreie Aufgehen in einer glatt laufenden Tätigkeit (Csikzentmihalyi, 1975/ 1999; Rheinberg, 2002), ist das Ziel der jetzigen Arbeit erreicht.

Natürlich kann man das verwendete Spiel Roboguard noch optimieren bzw. noch bessere Alternativen suchen. Mit dem jetzt vorliegenden Programm hat man aber schon einmal eine methodische Möglichkeit, mit der sich arbeiten lässt. So lassen sich jetzt je nach Forschungsinteresse unterschiedlichste Flow-Korrelate und -auswirkungen daraufhin untersuchen, ob sie (a) über Schwierigkeitsstufen hinweg dasselbe Muster zeigen, wie die Flow-Werte, und ob (b) innerhalb derselben Schwierigkeitsstufe individuelle Unterschiede des Flow-Erlebens in Zusammenhang stehen mit den interessierenden Korrelaten und Effekten.

Da die Tätigkeit konstant gehalten wird und zudem bis auf die rechte Hand keine Bewegung erforderlich ist, bietet sich auch die Möglichkeit, physiologische Indikatoren auf ihre Flow-Kovariation hin zu untersuchen. Das könnte zu einem entscheidenden Schritt auf dem Weg zu einer selbstberichtsfreien, quasi online-Erfassung von Flow-Zuständen werden.

In der jetzigen Arbeit haben wir exemplarisch den Flow-Einfluss einer zur Zeit kontrovers behandelten Personvariablen untersucht, nämlich den Einfluss der Zielorientierung. Natürlich müssen die jetzigen Befunde erst repliziert werden, bevor man weitere Schlüsse zieht. Dass diese Variable aber überhaupt mit Flow-Werten (Absorbiertheit 
unter geeigneten Anforderungsbedingungen) in nennenswertem Zusammenhang stand, ist genau betrachtet schon etwas erstaunlich. Kriterium war ein aktueller Zustand unter geeigneten Bedingungen eines Computerspiels (Flow bei optimaler Schwierigkeit). Prädiktor war aber eine generalisierte Tendenz, in Lernsituationen bestimmte Ziele anzustreben (dazuzulernen, Beifall zu erlangen, stolz auf sich sein zu können/Karriere zu machen). Wir schließen also von einer mehr oder weniger stabilen und allgemeinen Tendenz in relevanten Alltagssituationen auf einen besonderen Zustand in der hoch spezifischen Situation eines eher irrelevanten Computerspiels. Man hätte also gute Argumente gehabt, wenn man zwischen beiden Variablenebenen keine Beziehungen gefunden hätte.

Tatsächlich gab es aber einige Zusammenhänge und zwar nur dort, wo Flow-Erleben auftreten sollte, nämlich auf den optimalen Schwierigkeitsstufen. Auf den flow-ungeeigneten Schwierigkeitsstufen 2 (zu leicht) und 9 (zu schwer) findet man auch keine Zusammenhänge zwischen Zielorientierung und Flow.

Inhaltlich ergeben die Zusammenhänge ein interessantes und möglicherweise konsequenzenreiches Bild. Wir finden nirgendwo negative Zusammenhänge zwischen Zielorientierung und Flow! Erwartungswidrig zeigt sogar die Anerkennungssuche (approval seeking) mit $r=.29(p=.06)$ in der Tendenz einen positiven Zusammenhang mit dem FlowGesamtwert (s. Tabelle 2). Dass diese Zielorientierung zugleich auch mit dem Besorgnis-Wert der FKS korreliert, sollte nicht irritieren. Von Kletterdaten direkt aus der Felswand wissen wir, dass sich Flow und Besorgnis/negative Aktivierung keineswegs ausschließen, sofern die Bedrohung nicht allzu groß wird (Aellig, 2002). Letzteres ist bei unserem Computerspiel sicher nicht der Fall.

Sollten sich die jetzigen Befunde replizieren lassen, so könnte man für Nachfolgeexperimente versuchsweise die folgende Arbeitshypothese formulieren: Bei geeigneten Tätigkeitsanforderungen (voll auslastender Schwierigkeitsgrad, ständiger Zwang zur fortlaufenden und richtigen Reaktion, etc.) könnte es für den Flow-Zustand relativ 
belanglos werden, mit welcher Initialmotivation man in die fragliche Tätigkeit hinein geraten ist. Entscheidend könnte sein, dass man überhaupt in den unterbrechungsfreien und schnell laufenden Zyklus von eigenem Output und unmittelbarer Umweltantwort gerät, den man bei voller Mobilisierung eigener Ressourcen gerade noch gut beherrschen kann.

Befunde von Mannell, Zuzanek und Larsen (1988), wonach im Freizeitverhalten erwartungswidrig nicht etwa die locker entspannten Beschäftigungen, sondern gerade die „ernsthaften“, anstrengungsfordernden und/oder zwecküberlagerten Aktivitäten FlowZustände hervorriefen, wären mit unserer Arbeitshypothese durchaus vereinbar. Die Autoren vermuten, dass zur Herbeiführung des Flow-Zustandes ein allgemeines Trägheitsmoment von Menschen (Inertia Principle) überwunden werden müsse und das könne durch unterschiedlichste auch extrinsische Anreize geschehen. Entscheidend ist, dass man „tief“ genug in eine geeignete Tätigkeit hineingerät.

Damit wäre aus unserer Sicht allerdings nicht gesagt, dass die Motivationsqualität beim Flow nun gänzlich irrelevant sei. Insbesondere unter Alltagsbedingungen wird sie Einfluss darauf haben, unter welchen Bedingungen man sich auf die Kapazitätsanforderungen solcher flow-auslösenden Aktivitätszyklen überhaupt einlässt, wobei nach Mannell et al. (1988) die erlebte Entscheidungsfreiheit eine wichtige Rolle spielt. Analog zum RubikonModell (Heckhausen, 1987) wäre mit der Arbeitshypothese lediglich behauptet, dass Unterschiede der Initialmotivation mit Blick auf Flow dann keine Rolle mehr spielen, wenn man einmal über die passende Situation und Aufgabe hinreichend stark in die flow-typischen voll auslastenden Aktivitätszyklen hineingeraten ist.

Diese spekulative Arbeitshypothese ist theoretisch reizvoll und wäre mit Blick auf Anwendungsaspekte z. B. in Ausbildung und Beruf nicht ohne provokanten Witz. Man sollte versuchen, sie zu falsifizieren. 


\section{Literaturverzeichnis}

Aellig, S. (2003). Flow-Erleben und Wohlbefinden als Anreize für eine autotelische Tätigkeit. Über den Sinn des Unsinns. Eine Untersuchung mit der Experience Sampling Method (ESM) am Beispiel des Felskletterns. Dissertation, Universität Zürich.

Atkinson, J.W. (1957). Motivational determinants of risk-taking behavior. Psychological Review, 64, 359-372.

Barron, K.E. \& Harackiewicz, J.M. (2001). Achievement goals and optimal motivation: testing multiple goal modes. Journal of Personality and Social Psychology, 80, 706-722.

Csikszentmihalyi, M. (1975/1999). Beyond boredom and anxiety. San Francisco: Jossey-Bass. (deutsch: Das Flow-Erlebnis. Stuttgart: Klett-Cotta. 1999, 8. Auflage)

Csikszentmihalyi, M. \& Jackson, S.A. (2000). Flow im Sport. Der Schlüssel zur optimalen Erfahrung und Leistung. München: BLV.

Csikszentmihalyi, M. \& Larson, R. (1987). Validity and reliability of the Experience Sampling Method. Journal of Nervous and Mental Disease, 175, 529-536.

Csikszentmihalyi, M. \& LeFevre, J. (1989). Optimal experience in work and leisure. Journal of Personality and Social Psychology, 56, 815-822.

Dweck, C.S. \& Leggett, F.L. (1988). A social-cognitive approach to motivation and personality. Psychological Review, 95, 256-273.

Hayamizu, T. \& Weiner, B. (1991). A test of Dweck's model of achievement goals as related to perceptions of ability. Journal of Experimental Education, 59, 226-234.

Heckhausen, H. (1987). Perspektiven einer Psychologie des Wollens. In H. Heckhausen, P.M. Gollwitzer \& F.E. Weinert (Eds.), Jenseits des Rubikon (pp. 121-142). Berlin: Springer.

Heckhausen, H. (1989). Motivation und Handeln. Berlin: Springer.

Heckhausen, H., Schmalt, H.-D. \& Schneider, K. (1985). Achievement motivation in perspective. New York: Academic Press.

Jackson, S.A. \& Eklund, R.C. (2002). Assessing flow in physical activity: The Flow State Scale 2 and Dispositional Flow Scale 2. Journal of Sport and Exercise Psychology, 24, 133-150.

Jackson, S.A., Kimiecik, J.C., Ford, S.K. \& Marsh, H.W. (1998). Psychological correlates of flow in sport. Journal of Sport and Exercise Psychology, 20, 358-378.

Mannell, R.C., Zusanek, J. \& Larson, R. (1988). Leisure states and "Flow" Experiences: Testing perceived freedom and intrinsic motivation hypothesis. Journal of Leisure Research, 20, 289-304.

Moneta, G.B. \& Csikszentmihalyi, M. (1996). The effect of perceived challenges and skills on the quality of subjective experience. Journal of Personality, 64, 274-310.

Musch, J., Rahn, B. \& Lieberei, W. (2002). Bonner-Postkorb-Module (BPM). Göttingen: Hogrefe.

Nicholls, J.G. (1984). Achievement motivation: Conceptions of ability, subjective experience, task choice, and performance. Psychological Review, 91, 328-346. 
Novak, T.P., Hoffman, D.L. \& Young, Y.-F. (1998). Measuring the Flow construct in online environments: A structural modeling approach.

$h \mathrm{ttp}: / /$ ecommerce.vanderbilt.edu/papers/flow.construct/measuring_flow_construct.html.

Pfister, R. (2002). Flow im Alltag. Bern: Lang.

Remy, K. (2000). Entwicklung eines Fragebogens zum Flow-Erleben. Bielefeld:

Diplomarbeit. Fakultät für Psychologie und Sportwissenschaft.

Rheinberg, F. (2002). Freude am Kompetenzerwerb, Flow-Erleben und motivpassende Ziele. In M.v. Salisch (Ed.), Emotionale Kompetenz entwickeln (S. 179-206). Stuttgart: Kohlhammer.

Rheinberg, F., Engeser, S. \& Vollmeyer, R. (2002). Measuring components of flow: the FlowShort-Scale. Poster presented at the First International Positive Psychology Summit, 3.6.10.2002 inWashington, DC.

Rheinberg, F., Vollmeyer, R. \& Engeser, S. (2002). Die Erfassung des Flow-Erlebens. In J. Stiensmeier-Pelster \& F. Rheinberg (Eds.), Diagnostik von Selbstkonzept, Lernmotivation und Selbstregulation (S.261-279). Göttingen: Hogrefe.

Sansone, C. \& Harackiewicz, J.M. (2000). Intrinsic and extrinsic motivation. San Diego: Academic Press.

Schallberger, U. \& Pfister, R. (2001). Flow-Erleben in Arbeit und Freizeit. Eine Untersuchung zum Paradox der Arbeit mit der Experience Sampling Method. Zeitschrift für Arbeits- und Organisationspsycholgie, 45, 176-187.

Schubert, C. (1986). Motivationsanalysen zur Interaktion mit Computern. Diplomarbeit: Psychologisches Institut der Universität Heidelberg.

Stein, G.L., Kimiecik, J.C., Daniels, J.C. \& Jackson, S.A. (1995). Psychological antecedents of flow in recreational sport. Personality and Social Psychology Bulletin, 21, 125-135. 
Tabelle 1

Sechs Hauptkomponenten des Flow-Erlebens (nach Csikszentmihalyi, 1975; Rheinberg, 2002)

1) Passung von Fähigkeit und Anforderung. Trotz hoher Anforderung Erlebnis von Kontrolle. Gefühl, optimal beansprucht zu sein.

2) Handlungsanforderungen und Rückmeldungen sind völlig klar.

3) Der Handlungsablauf wird als glatt und flüssig erlebt. Alles läuft wie aus einer inneren Logik ( $\rightarrow$ Bezeichnung Flow).

4) Die Konzentration kommt wie von selbst. Das Stimulusfeld ist stark eingeengt.

5) Das Zeiterleben ist verändert, meist stark beschleunigt („Stunden vergehen wie Minuten“).

6) Verlust von Selbstreflexion und Bewertungskognitionen. Man ist nur noch Tätigkeit (,Verschmelzen“"von Selbst und Tun). 
Tabelle 2

Korrelationen zwischen Flow-Erleben (FKS) und Zielorientierung (AGT) bei optimal gepassten Anforderungen in einem Computerspiel

\begin{tabular}{l|cccc}
\hline \multicolumn{1}{c|}{$\begin{array}{c}\text { Dimension der } \\
\text { Zielorientierung (AGT) }\end{array}$} & $\begin{array}{c}\text { Flow } \\
\text { Gesamt }\end{array}$ & $\begin{array}{c}\text { Gutter, } \\
\text { automatischer } \\
\text { Verlauf }\end{array}$ & Absorbiertheit & Besorgnis \\
\hline Learning goal orientation & .23 & .04 & $.34^{*}$ & .16 \\
Performance advance goal & $.32 *$ & .14 & $.40^{* *}$ & .17 \\
Approval seeking goal & .29 & .25 & .23 & $.36^{*}$ \\
\hline
\end{tabular}

Legende: $* \mathrm{p}<.05 ; * * \mathrm{p}<.01$ 
Abb. 1: Schwierigkeitseinschätzung und Flow-Erleben (FKS) auf verschiedenen Schwierigkeitsstufen des Computerspiels Roboguard (benachbarte Schwierigkeitsstufen zusammengefasst)

Abb. 2: Mittlere Flow-Werte (FKS) in fünf Spieldurchgängen auf drei Anforderungsstufen

Abb. 3: Teilkomponenten der FKS auf drei Schwierigkeitsstufen (drei Durchgänge der Optimalschwierigkeit zusammengefasst) 

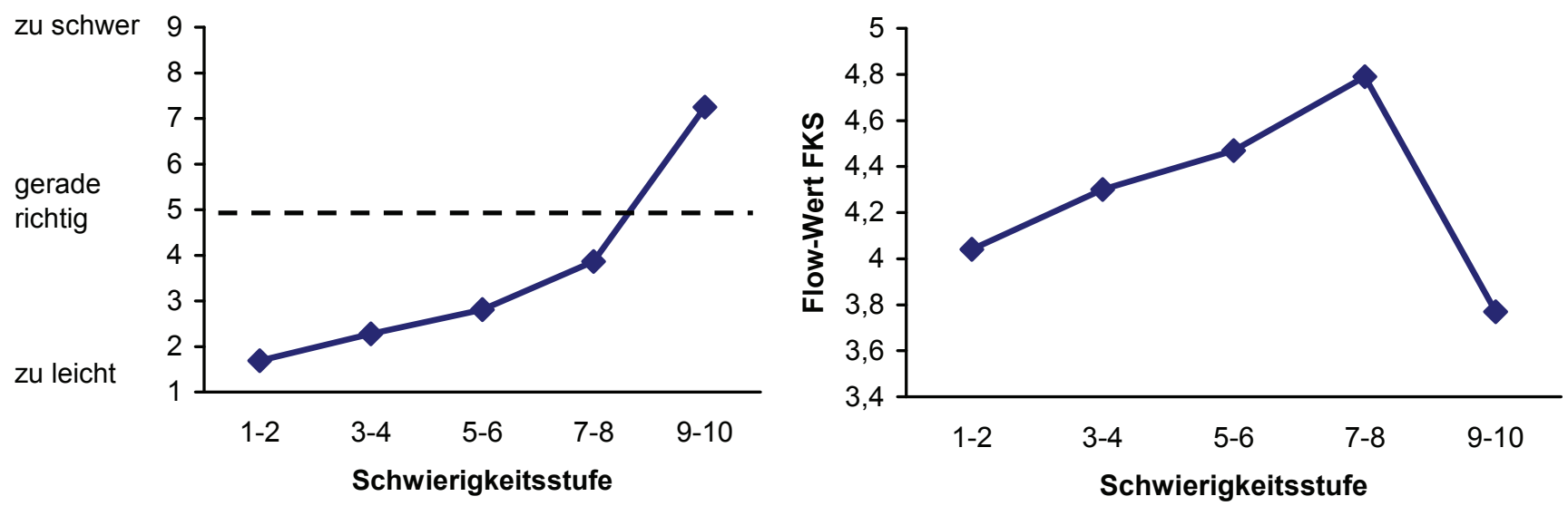


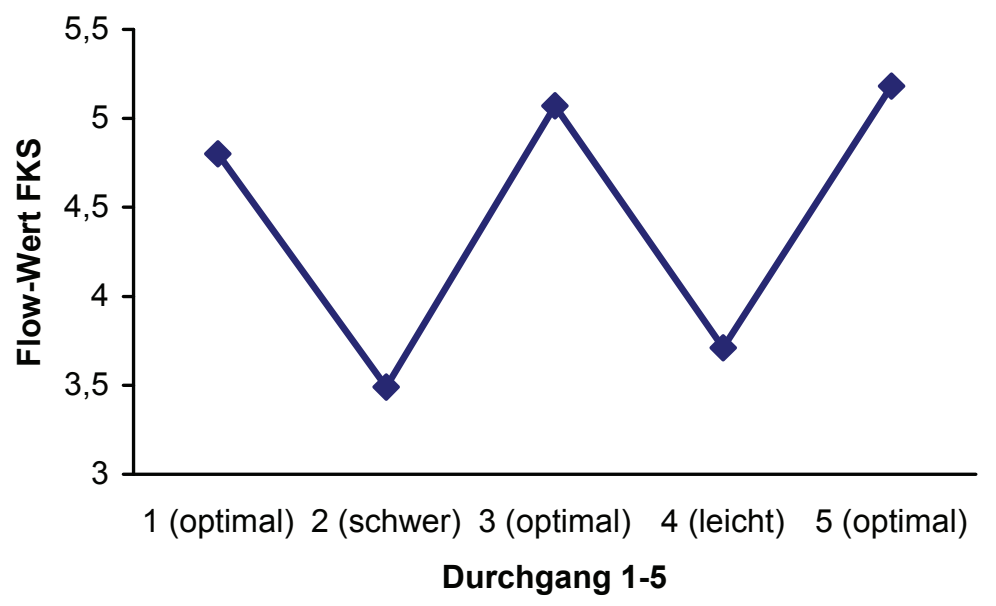




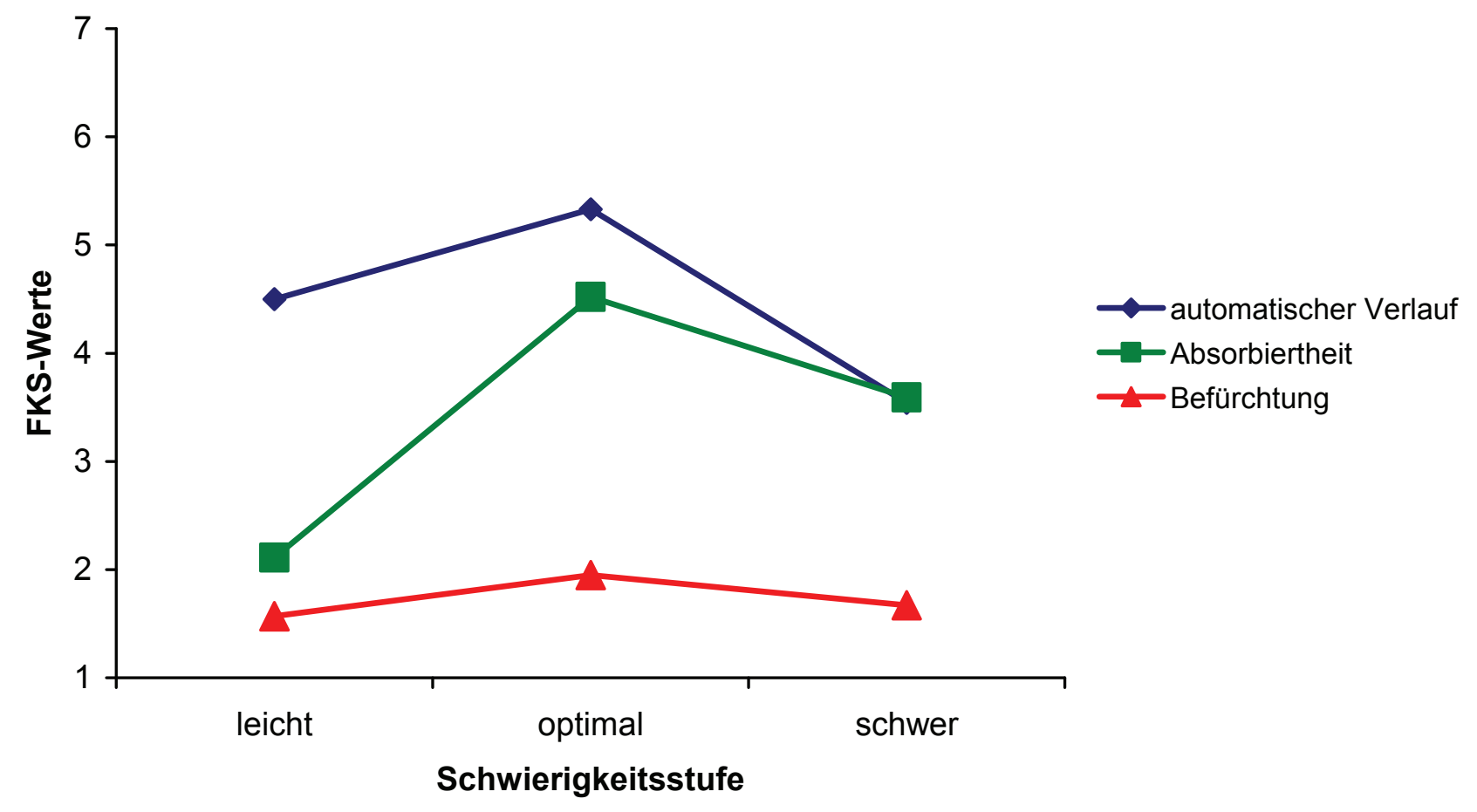

